\title{
A fuzzy methodology to alleviate information overload in eLearning
}

\author{
Fabio Aurelio D'Asaro ${ }^{1}$ Valerio Perticone ${ }^{2}$ Marco Elio Tabacchi ${ }^{3}$ \\ 1,2 DMI, Università degli Studi di Palermo (Italy) \\ ${ }^{3}$ DMI, Università degli Studi di Palermo and Istituto Nazionale di Ricerche Demopolis (Italy)
}

\begin{abstract}
Some aspects of eLearning experience can be enhanced in a very natural way by using the basic tools offered by fuzzy logic. As a matter of example, consider the uncontrolled growth of information produced in a collaborative-oriented context, in which each participant (e.g. students, teachers) is able to insert and share new contents (e.g. comments, texts) concerning a university course. All the incrementally added pieces of information can be evaluated in several ways: by the intervention of a "dictator" (e.g. the teacher), using a rating form, or even according to the frequency of access. As contents rapidly become unusable for the effects of information overload, basic tools of fuzzy logic such as membership functions and measures of fuzziness can help to distinguish between relevant and trivial content, without thereby canceling any contribution. This very same idea can of course also be applied to different contexts.
\end{abstract}

Keywords: eLearning, info overload, Fuzziness.

\section{Introduction}

The aim of the present paper is to outline a path which allows to save possible comments and revisions to a presentation of certain subjects so that all the past history of the teaching of a certain topic could be never lost.

The idea for this is the most trivial one: "add every comment to every notion when this happens to be done in a incremental way". The simplicity and triviality of this procedure is also at the origin of its main defect: the information can grow in an incontrollable way putting all the information at the same level without distinguishing between a very meaningful and a trivial information.

We propose to use the basic feature of fuzzy sets (graded membership) to classify and discriminate among all the incremental clarifications provided to a given text (we limit ourselves to the most basic elements of the notion of fuzzy set). One plans finally to provide a quantitative evaluation of how much the additional information can be easily distinguished and divided between interesting/not interesting by using the theory of "measures of fuzziness". Those fuzzy sets which have a low index are good candidate for easily eliminating the uninteresting comments, those with a high index show that the comments are more graded and a solution can be provided by more complex procedures. It could be also the case that a real major complexity of the topic in question emerges naturally in this way.

This paper is organized as follows: in the next section we shall fix the basic notation and terminology used; some measures of fuzziness will also be briefly introduced. In the third section we shall shortly survey the state of the art, listing some recent results and the tools used in literature in the fields of collaborative eLearning and evaluation of user-generated contents. In the fourth section we will describe our approach to the problem through the use of examples. The last section presents some conclusion and shows some possible developments of this work.

\section{Fuzzy logic}

In the present section we shall present and fix the basic notation that will be used later.

\subsection{Notation and terminology}

A fuzzy set $A$ in the universe of discourse $U$ is usually determined by his membership function

$$
\mu_{A}: U \rightarrow[0,1]
$$

that maps each element $x \in U$ to its degree of membership to the set $A . \mathscr{F}(U)=\{\mu$ such that $\mu: U \rightarrow$ $[0,1]\}$ is the set whose elements are all the possible membership functions of fuzzy sets in the universe U.

The support of a fuzzy set $A$ is defined as

$$
s(A)=\left\{x \in U: \mu_{A}(x)>0\right\}
$$

and $A$ is called a finite fuzzy set if its support is finite. Its crisp part or kernel is

$$
c(A)=\left\{x \in U: \mu_{A}(x)=1\right\}
$$

and, finally, its $\alpha$-cuts are

$$
A^{\alpha}=\left\{x \in U: \mu_{A}(x) \geq \alpha\right\}
$$

for $\alpha \in[0,1]$. 
A classical set $B$ can be considered as a fuzzy set described by his characteristic function and, viceversa, a fuzzy set having a membership function whose range is $\{0,1\}$ is called a crisp set.

The original way of defining union and intersection between two sets $A$ and $B$ and the complement of a set $A$ is the following:

$$
\begin{gathered}
\mu_{A \cup B}(x)=\max \left(\mu_{A}(x), \mu_{B}(x)\right) \\
\mu_{A \cap B}(x)=\min \left(\mu_{A}(x), \mu_{B}(x)\right) \\
\mu_{A^{C}}(x)=1-\mu_{A}(x)
\end{gathered}
$$

These are the original definitions by Zadeh [1]. Let us remember that in the same paper, Zadeh presented also alternative possible ways of defining such operators and that subsequently there has been a huge and profound work for introducing and studying generalized connectives (see $[2,3]$ for a more comprehensive survey); however, in what follows it suffices to consider the basic definitions just introduced.

\subsection{Defuzzification}

In most applications, one needs to infer precise numerical values from imprecise statements represented by means of fuzzy sets. Among many possibilities, we shall here present only some basic methods: the centre of gravity method (COG), and the maxima-based methods.

The COG method obtains a crisp value $y$ by means of a weighted average:

$$
y=\sum_{x \in U}\left(x \cdot \frac{\mu_{A}(x)}{\sum_{x \in U} \mu_{A}(x)}\right)
$$

while maxima methods are based on various statistical features of the set of those $x$ such that $\mu_{A}(x)$ is maximal. For example, the mean of maxima method (MOM) is defined as follows:

$$
y=\sum_{x \in M} \frac{x}{|M|}
$$

in which $M=\left\{x: \mu_{A}(x)=\max _{x \in U} \mu_{A}(x)\right\}$ and $|M|$ is its cardinality. In a similar way it is possible to define methods such as first of maxima (FOM), last of maxima (LOM) and median/centre of maxima (COM).

See Figure 1 to get an instantaneous description of these methods.

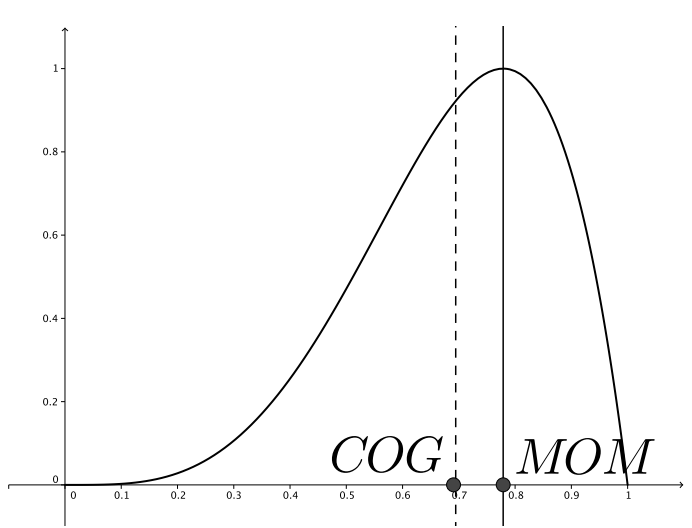

Figure 1: Centre of gravity (COG) and mean of maxima (MOM) of a fuzzy set

\subsection{Measures of fuzziness}

The idea behind a fuzzy set is to specify how much is certain that a given element belongs to a given set. It is therefore natural to ask for the degree of fuzziness of a set, or, to put it another way, the global difficulty of deciding which elements belongs to a set. The answer is given by the theory of "measures of fuzziness", firstly proposed in [4]. For a survey of this theory see [5]. A measure of fuzziness is a nonnegative mapping $d: \mathscr{F}(U) \rightarrow[0,+\infty[$ satisfying at least the following conditions:

- Sharpness: $d(A)=0$ if and only if $A$ is a crisp set

- Maximality: $d(A)$ is maximal when $\mu_{A}(x)=$ $1 / 2$ for each $x \in U$

- Resolution: $d(A *) \leq d(A)$, with $A *$ being a sharpened version of $A$, that is: $\mu_{A *}(x) \leq$ $\mu_{A}(x)$ when $\mu_{A}(x) \leq 1 / 2$ and $\mu_{A *}(x) \geq \mu_{A}(x)$ when $\mu_{A}(x) \geq 1 / 2$.

Other reasonable conditions that can be adopted are:

- Simmetry: $d(A)=d\left(A^{C}\right)$

- Valuation: $d(A \cup B)+d(A \cap B)=d(A)+d(B)$

Ebanks has shown that, by adding a further technical condition (called generalized additivity), the measure of fuzziness provided by $d(A)=$ $\sum_{x \in U} \mu_{A}(x)\left|1-\mu_{A}(x)\right|$ is unique [6].

The following are some particular measures of fuzziness $d$ given a finite universe $U=$ $\left\{x_{1}, x_{2}, \ldots, x_{n}\right\}$ :

- Fuzzy Entropy [4]:

$$
d(A)=\frac{1}{n} \sum_{x \in U} S\left(\mu_{A}(x)\right)
$$

where $S(x)=-x \ln x-(1-x) \ln (1-x)$ is the Shannon function. The value $\frac{1}{n}$ normalizes $d(A)$ into the range $[0,1]$ if the logarithm in base 2 is taken. 
- Linear index of fuzziness [7]:

$$
d(A)=\frac{2}{n} \sum_{x \in U}\left|\mu_{A}(x)-\mu_{A^{0.5}}(x)\right|
$$

in which $\mu_{A^{0.5}}$ is the membership function of the 0.5 -cut of $A$. Ultimately, $d(A)$ is the distance between $A$ and its closest crisp version $A^{0.5}$

- Quadratic index of fuzziness [7]:

$$
d(A)=\sqrt{\frac{4}{n} \sum_{x \in U}\left[\mu_{A}(x)-\mu_{A^{0.5}}(x)\right]^{2}}
$$

\section{State of the art}

\subsection{Collaborative eLearning}

The use of electronic instruments in the field of teaching has recently gained attention for his proven capacity of increasing the fruition of contents and the learning quality $[8,9]$. This mixture of technology and learning is usually known as eLearning. Its applications range from the simple use of computeraided technologies during lessons to hybrid learning and pure online learning.

Recently eLearning, supported by the new instruments that are being available as internet and ICT grow up (e.g. wikis, forums, mind-maps, social networks...), is moving towards a new - more collaborative - way of handling and communicating knowledge.

In fact, statistical evidence seem to prove that the "new millennium learner" wants/needs to be an active, collaborative part of the learning process $[10,11]$. This has progressively led to a new approach that is called Computer-supported collaborative learning (CSCL) [12].

In such a collaborative context each user is generally able to add new contents, and/or remove and modify existing ones (as it happens, for example, on Wikipedia). For this kind of interaction to be effective, several strategies and organizational patterns are to be applied, e.g. synchronous or asynchronous writing, parallel or single-writing $[13,14,15]$. Furthermore, in order to gain knowledge one needs to fetch useful information [16] among a great amount of data produced by many different sources, a phenomenon known as information overload, while at the same time trying to build a single and coherent shared source of knowledge.

As we shall see in the following section, this very problem - that is the one we are mostly concerned with - has yet been addressed in several ways involving (automated) evaluation of contents.

\subsection{Evaluation}

Evaluation of content has been recently studied for its wide range of applicability. As a matter of example, evaluation of books, movies and other commercial products has a great importance in business

\begin{tabular}{|r|c|c|c|c|c|}
\cline { 2 - 6 } \multicolumn{1}{c|}{} & U1 & U2 & U3 & Entropy & Mean \\
\hline simple & 1 & 0.7 & 0.6 & $\mathbf{0 . 6 2}$ & $\mathbf{0 . 7 7}$ \\
\hline correct & 0.9 & 1 & 0.9 & $\mathbf{0 . 3}$ & $\mathbf{0 . 9 3}$ \\
\hline clear & 0.8 & 0.8 & 0.5 & $\mathbf{0 . 8 1}$ & $\mathbf{0 . 7}$ \\
\hline interesting & 1 & 0.7 & 0.8 & $\mathbf{0 . 5 3}$ & $\mathbf{0 . 8 3}$ \\
\hline illustrative & 0.9 & 0 & 0 & $\mathbf{0 . 3}$ & $\mathbf{0 . 3 3}$ \\
\hline
\end{tabular}

Table 1: From linguistic attribution to defuzzification. We have used (10) as our Entropy function.

and e-commerce [17, 18]. This kind of evaluation is mainly based on users' ratings (e.g. Likert-like scales [19], star systems [17]) and hence on their previous experiences. The typical aims of this systems employed in evaluation are to use information about customers to suggest personalized recommendations [20, 18] and to assess a second party's reliability $[21,22]$. However, since we are here concerned with university courses, this approach is not suitable for our purposes because a significative portion of our audience is renewed periodically and moreover we have completely different intents. For this reason, we shall exclusively concentrate on evaluation of contents.

Having a qualitative evaluation of a single content in an unstructured collection of data can be useful in order to discriminate between related pieces of information with different quality [23, 24].

The vast use of wiki technologies (e.g. Wikipedia) allowed researchers to study evaluation of usergenerated contents $[25,26]$. A series of studies was conducted to rate wiki pages in a fully manual way $[27,28]$; more recently, some instruments like Wikipedia's "Article Feedback Tool" [29] try to assess the quality of a page in a e-commerce-like fashion.

\section{Our model}

We set ourselves in the case in which all the users are able to incrementally add contents (that are related to the same subject or topic) in an uncontrolled way. Our main aim is to describe a CSCL platform for eLearning in which fuzzy methodologies are used to rise relevant pieces of information above less considerable ones. The basic steps of the process are essentially the following:

1. Contents' features are evaluated.

2. Evaluations are defuzzified.

3. Fuzzy measures and further statistics are extracted and exploited to identify and isolate most interesting pieces of information.

Let us now consider the example of a university course in which students are invited to add different explanations for a given topic, which in our example is represented by the Productivity of a Turing Machine (TM), a concept connected to the well-known 
busy beaver problem. Three users of the platform try to explain the same topic in their own way:

\section{User 1:}

Given a TM M, we define its productivity as the number of " 1 " on its tape at the end of a correct computation (i.e. the machine halts correctly) and with the further tie that $M$ has no input symbol on its tape when the computation begins. If the computation does not halt, then the productivity of the given machine is 0 . For example, consider the 2-state-TM described in the following figure:

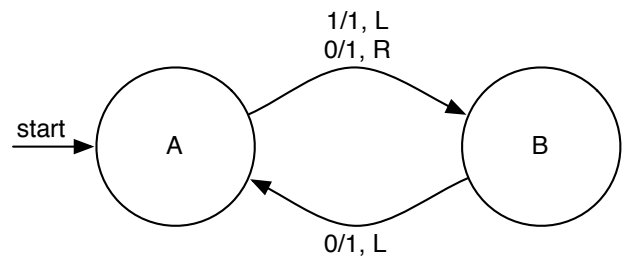

its productivity is 4 , as we can see from a complete computation:

$$
\begin{aligned}
& \begin{array}{llllllll}
0 & 0 & 0 & 0 A O & 0 & 0 & 0 & 0
\end{array} \\
& \begin{array}{lllllllll}
0 & 0 & 0 & 0 & 1 \mathrm{~B} 0 & 0 & 0 & 0
\end{array} \\
& \begin{array}{llllllll}
0 & 0 & 0 & 0 A 1 & 1 & 0 & 0 & 0
\end{array} \\
& \begin{array}{llllllll}
0 & 0 & O B O & 1 & 1 & 0 & 0 & 0
\end{array} \\
& \begin{array}{llllllll}
0 & O A O & 1 & 1 & 1 & 0 & 0 & 0
\end{array} \\
& \begin{array}{llllllll}
0 & 0 & 1 B 1 & 1 & 1 & 0 & 0 & 0
\end{array}
\end{aligned}
$$

Furthermore, it is easy to see that the machine depicted below never halts; thus, its productivity is 0 .

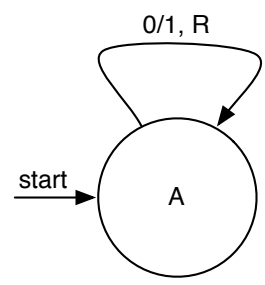

It is now possible to define a productivity function as follows:

$$
p(n)=\max _{M \in A_{n}}\{\text { productivity of } M\}
$$

in which $A_{n}$ is the well-defined set of all TMs having exactly $n$ states. As we'll prove, this function is not computable.

\section{User 2:}

We define productivity of a given TM M as the number of " 1 " that lie on the tape at the end of M's computation starting with blank tape. If the machine does not halt we'll say that its productivity equals 0 .

Let us now define the function $\mathrm{p}(\mathrm{n})$ as the $\max$ imum productivity that a n-state machine can reach. One can now ask: is it possible to build a TM that calulates $p(n)$ for each value of $\mathrm{n}$ ? The answer - as we can expect from some previously proved theorems - is no: this problem is unsolvable.

\section{User 3:}

The productivity $\sigma(M)$ of a TM M is the number of ones left on its tape at the end of a computation starting with no symbols on the input tape. We have $\sigma(M)=0$ in the following two cases:

1. $M$ does not reach a final state

2. $M$ does not halt

Let $p(n): \mathbb{N} \rightarrow \mathbb{N}$ be the productivty function defined as $p(n)=$ $\max \left(\sigma\left(M_{1, n}\right), \sigma\left(M_{2, n}\right), \ldots, \sigma\left(M_{k_{n}, n}\right)\right)$ where $M_{i, n}$ for $1 \leq i \leq k_{n}$ is a Turing Machine having $\mathrm{n}$ states, and $k_{n}$ is the number of all these machines. It is possible to show that $p(n)$ is not computable.

Various features of these contents (e.g. "simplicity", "correctness", "clarity", etc.) should be rated. To this end, we can choose between several evaluation methods:

- A "dictator" (e.g., in this case, the teacher) or a group of experts impose its evaluation from above, using natural language.

- Users and contributors alike rate their agreement on the possession of specific features, using for example a five-point Likert scale.

- Some statistical parameters are taken in consideration, such as page hits, viewing time or length of users' session.

Firstly we interpret each judgement as a fuzzy set, so that we can use a a rather straightforward Computing With Words methodology [30, 31] and a defuzzification method (e.g. one of the methods described in Subsection 2.2) to infer numerical values from qualitative statements (see Figure 2 for an example). In our example, the results of this step are summarized in Table 1, where U1, U2, U3 respectively stand for User 1, User 2, User 3. Obtained numerical values can be regarded as the degree of membership of each contribution to a given feature - e.g., the degree up to which third user's contribution belongs to the set of "simple" contents is 0.6. It is now possible to determine the quality of each feature extracting fuzzy measures and some sensible 
statistical measures.

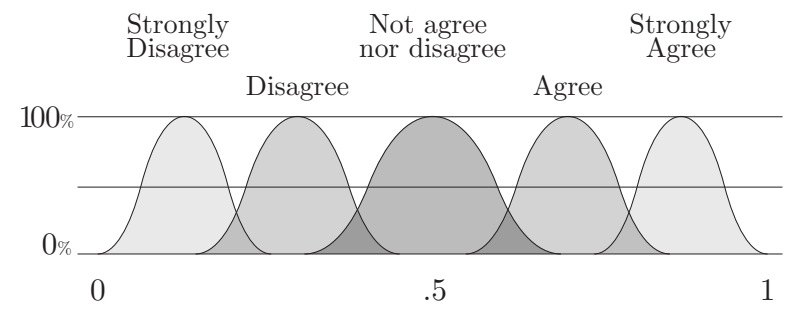

Figure 2: Fuzzy Likert scale.

In particular, we want to stress the following point: an high index of fuzzy entropy (or, in general, of any appropriate fuzzy measure - in Table 1 we simply have used Equation 10; it is possible however to consider different measures, such as those described in Equations 11 and 12 to obtain more pregnant, better results) naturally suggests that, to a certain extent, the specified feature is controversial and more complex to handle; conversely, a low index of fuzzy entropy indicates that the particular feature is solid as it is, and good and bad contributions to the topic can be easily distinguished. Ideally, as the course is updated from year to year, the entropy should converge to zero. In addition, it is possible to consider some appropriate statistical measures - e.g. mean, variance - to get a further characterization of the considered features.

In our view, these indications are useful to have a better understanding of the present state of the course, its strengths and its weaknesses; moreover, most relevant contributions can be immediately identified and used to make the course's entropy convergency faster. In fact, we can infer from Table 1 that the most problematic aspects related to the considered lesson are "clarity" and "simplicity" (fuzzy entropy is significantly greater than 0.5 ), while "correctness" is a better assessed aspect having a low fuzzy entropy index; it is important to note here that despite the "illustrative" parameter has a low mean, the entropy of the corresponding set is lower than 0.5 , indicating that the corresponding feature is well assessed - in fact, contributions to the topic are only "very illustrative" or "not illustrative at all"; the teacher can then consider improving the course by refining the so highlighted aspects. Furthermore, it is possible to exploit the fuzzy set representation to browse relevant contents through the use of $\alpha$-cuts. In our example, simple $0.8=\{U$ ser 3$\}$, indicating that the third user's contribution can be taken into account for its simplicity.

As a further detail, we want to highlight the fact that especially in the initial stages of the course, only a few evaluated comments could be available. In order to avoid making inferences from data that is still not significant, a (possibly fuzzy) threshold can be included.

\section{Conclusions}

In this contribution we have discussed contents and evaluations as fuzzy sets. As we have shown in the previous section, this approach lead to a simplified treatment of the information overload due to the activity of many users contributing to incrementally build a single source of knowledge, without the need to eliminate any content. Even better, this kind of representation allows to highlight and point out which features of the considered topic are solid and well assessed, and also gives us a way of determine which topics are the most controversial and complex, through the use of fuzzy measures.

It is remarkable that this methodology can be applied not only to university courses and classes, but also to situations where one needs to evaluate a great amount of data originated from different sources - e.g. reviews and criticism of books, films, works of art. In fact, as we have already seen in Subsection 2.3, the theory of "measures of fuzziness" allows to define many different measures of entropy of a fuzzy set, and such measures can be applied to a whole host of diverse disciplines $[32,33,34,35,36,37,38]$.

In future works we shall implement the described platform in a web-based application in order to apply and test this methodology to real cases such as university courses, aiming to collect data and evidence about its effectiveness in a comparative study.

\section{References}

[1] Lotfi A. Zadeh. Fuzzy sets. Information and Control, 8:338-353, 1965.

[2] Enric Trillas. Conjuntos borrosos. Vicens vives, 1980.

[3] Petr Hájek. Metamathematics of fuzzy logic, volume 4. Springer, 1998.

[4] Aldo De Luca and Settimo Termini. A definition of a nonprobabilistic entropy in the setting of fuzzy sets theory. Information and Control, 20(4):301 - 312, 1972 .

[5] Aldo De Luca and Settimo Termini. Entropy measures in fuzzy set theory. In Madhusudan G. Singh, editor, Systems and Control Encyclopedia, pages 1467-1473. Pergamon Press, 1988.

[6] Bruce R. Ebanks. On measures of fuzziness and their representations. J. Math. Anal. and Appl., 94:24-37, 1983.

[7] Arnold Kaufmann. Introduction to the theory of fuzzy subsets. Introduction to the Theory of Fuzzy Subsets. Academic Press, 1975.

[8] Dongsong Zhang, Lina Zhou, Robert O. Briggs, and Jay F. Nunamaker, Jr. Instructional video in e-learning: Assessing the impact of interactive video on learning effectiveness. Inf. Manage., 43(1):15-27, January 2006. 
[9] Shu-Sheng Liaw. Investigating students' perceived satisfaction, behavioral intention, and effectiveness of e-learning: A case study of the blackboard system. Computers \& Education, 51(2):864-873, 2008.

[10] Francesc Pedró. The new millennium learners: Challenging our views on ict and learning. IDB Publications 9228, Inter-American Development Bank, May 2006.

[11] OECD. New millennium learners. initial findings on the effects of digital technologies on school-age learners. Technical report, OECD/CERI, 2008.

[12] Gerry Stahl, Timothy Koschmann, and Dan Suthers. Computer-supported collaborative learning: An historical perspective. In Cambridge University Press, editor, The Cambridge Handbook of the Learning Sciences, pages 409 426. R. K. Sawyer, 2006.

[13] Cindy E. Hmelo-Silver. Analyzing collaborative knowledge construction: multiple methods for integrated understanding. Computers \& Education, 41(4):397-420, 2003.

[14] Paul Benjamin Lowry, Aaron Curtis, and Michelle René Lowry. Building a taxonomy and nomenclature of collaborative writing to improve interdisciplinary research and practice. Journal of Business Communication, 41(1):6699, January 2004.

[15] Javier Onrubia and Anna Engel. Strategies for collaborative writing and phases of knowledge construction in cscl environments. Computers Es Education, 53(4):1256-1265, 2009.

[16] Robin S. Poston and Cheri Speier. Effective use of knowledge management systems: a process model of content ratings and credibility indicators. MIS Q., 29(2):221-244, June 2005.

[17] Susan M. Mudambi and David Schuff. What makes a helpful online review? a study of customer reviews on amazon.com. MIS Q., 34(1):185-200, March 2010.

[18] Chumki Basu, Chumki Basu, Haym Hirsh, and William" Cohen. Recommendation as classification: Using social and content-based information in recommendation. In Proceedings of the fifteenth national conference on artificial intelligence, pages 714-720, 1998.

[19] Rensis Likert. A technique for the measurement of attitudes. Archives of Psychology, 22(140):1-55, 1932.

[20] Ulrike Gretzel and Daniel R. Fesenmaier. Persuasiveness of preference elicitation processes in destination recommendation systems. In Andrew J. Frew, editor, Enter, pages 194-204. Springer, 2005.

[21] Paul Resnick, Paul Resnick, Richard Zeckhauser, John Swanson, and Kate" Lockwood. The value of reputation on ebay: A controlled experiment. Experimental Economics, 9:79 101, 2003.
[22] Sulin Ba. Establishing online trust through a community responsibility system. Decision Support Systems, 31(3):323 - 336, 2001.

[23] Lawrence Page, Lawrence Page, Sergey Brin, Rajeev Motwani, and Terry" Winograd. The pagerank citation ranking: Bringing order to the web. Stanford Infolab, page 17, 1999.

[24] Yanhong Li. Toward a qualitative search engine. IEEE Internet Computing, 2(4):24-29, July 1998.

[25] Yeomans Wheeler. The good, the bad and the wiki: Evaluating student-generated content for collaborative learning. British Journal of Educational Technology, 39(6):987-995, 2008.

[26] B. Thomas Adler and Luca de Alfaro. A content-driven reputation system for the wikipedia. In Proceedings of the 16th international conference on World Wide Web, WWW '07, pages 261-270, New York, NY, USA, 2007. ACM.

[27] Aniket Kittur, E Chi, and Bongwon Suh. Crowdsourcing for usability: Using micro-task markets for rapid, remote, and low-cost user measurements. Proc. CHI 2008, 2008.

[28] Aniket Kittur and Robert E. Kraut. Harnessing the wisdom of crowds in wikipedia: quality through coordination. In Proceedings of the 2008 ACM conference on Computer supported cooperative work, CSCW '08, pages 37-46, New York, NY, USA, 2008. ACM.

[29] Aaron Halfaker, Oliver Keyes, and Dario Taraborelli. Making peripheral participation legitimate: reader engagement experiments in wikipedia. In Proceedings of the 2013 conference on Computer supported cooperative work, CSCW '13, pages 849-860, New York, NY, USA, 2013. ACM.

[30] Lofti A. Zadeh. Fuzzy logic = computing with words. Fuzzy Systems, IEEE Transactions on, 4(2):103 -111, 1996.

[31] Lotfi A. Zadeh. From computing with numbers to computing with words - from manipulation of measurements to manipulation of perceptions. International Journal of Applied Mathematics and Computer Science, 12:307324, 2002.

[32] Maurizio Cardaci, Vito Di Gesù, Maria Petrou, and Marco Tabacchi. On the evaluation of images complexity: A fuzzy approach. In Isabelle Bloch, Alfredo Petrosino, and Andrea Tettamanzi, editors, Fuzzy Logic and Applications, volume 3849 of Lecture Notes in Computer Science, pages 305-311. Springer Berlin / Heidelberg, 2006.

[33] Baingio Pinna and Marco Tabacchi. A fuzzy approach to the role of symmetry in shape formation: The illusion of the scalene triangle. In Vito Di Gesù, Sankar Pal, and Alfredo Petrosino, editors, Fuzzy Logic and Applications, volume 5571 of Lecture Notes in 
Artificial Intelligence, pages 197-204. Springer Berlin-Heidelberg, 2009.

[34] Marco Elio Tabacchi and Settimo Termini. Measures of fuzziness and information: some challenges from reflections on aesthetic experience. In Proceedings of WConSC 2011, 2011.

[35] Marco Elio Tabacchi and Settimo Termini. Birkhoff's aesthetics, arnheim's entropy. some remarks on complexity and fuzzy entropy in arts. 2011.

[36] Marco Elio Tabacchi and Settimo Termini. Varieties of vagueness, fuzziness and a few foundational (and ontological) questions. In Proceedings of EusFLAT 2011, Advances in Intelligent Systems Research, pages 578-583. Atlantis Press, 2011.

[37] Marco Elio Tabacchi and Settimo Termini. A few remarks on the roots of fuzziness measures. In Salvatore Greco, Bernadette Bouchon-Meunier, Giulianella Coletti, Mario Fedrizzi, Benedetto Matarazzo, and RonaldR. Yager, editors, Advances in Computational Intelligence, volume 298 of Communications in Computer and Information Science, pages 62 67. Springer Berlin Heidelberg, 2012.

[38] Barbara Caci, Maurizio Cardaci, and Marco Tabacchi. Facebook as a small world: a topological hypothesis. Social Network Analysis and Mining, 2:163-167, 2012. 10.1007/s13278-0110042-8. 